\title{
X Band Phased Array Design for Radar Application
}

\author{
J.J. Cárdenas-Triana , I.D. Duarte Brito , J.M. Fernández-González , M. Sierra-Castañer
}

\begin{abstract}
The design process and simulation of a phased array antenna for a coastal surveillance radar application in $X$ band $(8.8-9.6 \mathbf{G H z})$ is presented. A printed folded dipole is chosen as radiating element due to its feasible behaviour and fabrication facilities. The designed array consists of 5 rows of 144 elements each one, achieving a total dimensions of $260 \mathrm{~cm}$ width and 11.5 cm heigth.
\end{abstract}

Index Terms-antenna design, phased array, radar antennas. electronic scanning.

\section{INTRODUCTION}

In radar systems, the antenna has the function to transmit the electromagnetic energy through the medium and collect the reflected energy of a distant target. In order to do the described process in an efficient way, the device should have an appropriate impedance matching, high gain, low side-lobes level and guarantee a precise angular resolution enough for the application. For that reason, it is almost mandatory to use large aperture antennas, commonly reflectors and large arrays to achieve the mentioned specifications. Antenna arrays have the capability to steer their beam electronically, avoiding the mechanical wear and making possible a dynamic and efficient beam control, one of the fundamental characteristics of modern radar systems. The phased arrays operation is primarily based on introducing an appropriate phase difference between the adjacent array elements, so the desired beam tilt is achieved.

Historically, the phased array technology has been mainly impulsed by satellite surveillance and ballistic missile defense applications, although with the accelerated development of solid state active elements, the MIMIC ( Millimeter and $\mathrm{Mi}$ crowave Monolithic Integrated Circuits) and industry growth, it is possible to use this technology on ground based and airborne radar applications as well. Nowadays, new fields of interest have emerged, as photonic beam forming, microelectromechanical phase shifters and space-time adaptive processing arrays, allowing the progress of phase array technology and its adaptation to the new challenges that civilian and military industry impose[1].

A phased array antenna design for a coastal surveillance radar application in $\mathrm{X}$ band $(8.8-9.6 \mathrm{GHz})$ is presented. The design specifications are based on the IALA (International Association of Marine Aids To Navigation and Lighthouse Authorities) recommendations for VTS (Vessel Traffic Services) systems and a comparison of diverse coastal surveillance radar products available in the market. The design specifications are listed on table I.
TABLE I: DESIGN SPECIFICATIONS.

\begin{tabular}{|c|c|}
\hline Parameter & Values \\
\hline \hline Scanning & Electronic \\
\hline Azimuthal Coverage & $90^{\circ}$ \\
\hline Radiation Pattern & Fan \\
\hline$\theta_{-3 d B}$ Azimuth & $0.7^{\circ}-0.8^{\circ}$ \\
\hline$\theta_{-3 d B}$ Elevation & $20^{\circ}-25^{\circ}$ \\
\hline Operation Bandwidth & $8.8-9.6 \mathrm{GHz}$ \\
\hline Polarization & Linear Horizontal \\
\hline Gain & $>30 d B$ \\
\hline Side Lobes Ratio & $>25 d B$ \\
\hline Max Input Power & $5 W$ \\
\hline
\end{tabular}

The remaining part of the article is organized as follows, in the second section the array design is presented, determining the distance between adjacent elements and rows, the amplitude weighting and additional design consideration effects. In the third section the modeling and simulation of the radiating element is described. In the fourth section the distribution network design is detailed. Finally, in the fifth section the conclusions of the work are presented.

\section{ARRAY DESIGN}

The antenna power gain $(G)$ and the half power beamwidth $\left(\theta_{-3 d B}\right)$ are related to the directivity and the effective antenna aperture, therefore it is necessary to achieve a suitable combination of two main parameters, the radiating elements separation $(d)$, and the number of elements $(N)$, avoiding the grating lobes appearance. It is important to note that, the largest numbers of elements, the narrowest is the beam, but the feeding network design will be more complex and also the losses will be higher. Then it is necessary to find a compromise between the number of elements and complexity of the feeding network. The chosen values in order to fulfill the specifications are summarized in table II.

TABLE II: ARRAY PARAMETERS.

\begin{tabular}{|c|c|c|}
\hline & Rows & Elements \\
\hline \hline Number $(N)$ & 5 & 144 \\
\hline Separation $(d)$ & $0.7 \lambda$ & $0.54 \lambda$ \\
\hline
\end{tabular}

For radar applications it is important to have control over the side lobes level, therefore it is necessary to use an amplitude weighting technique to reduce it according to design specifications, however if the side lobes level is reduced a gain loss and enlargement of the half power beamwidth is obtained. In order to achieve a $S L R$ (Side Lobe Ratio) higher than $25 d B$, the Taylor's window is chosen, due to $\theta_{-3 d B}$ parameter 


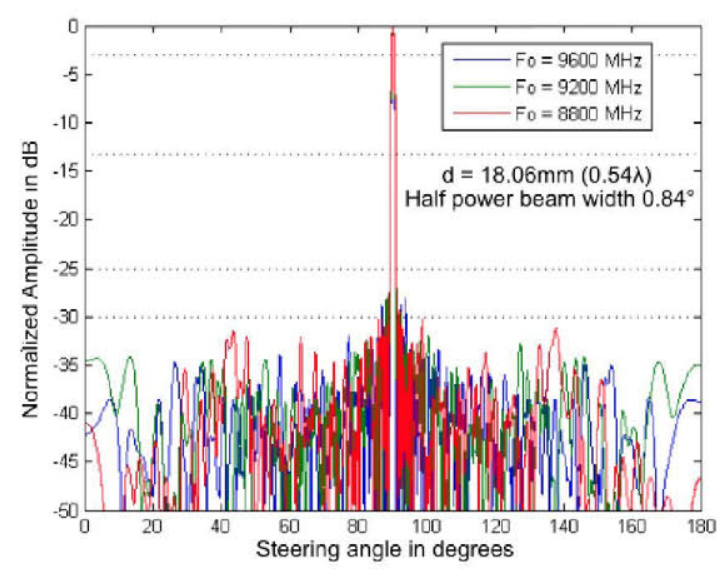

(a)

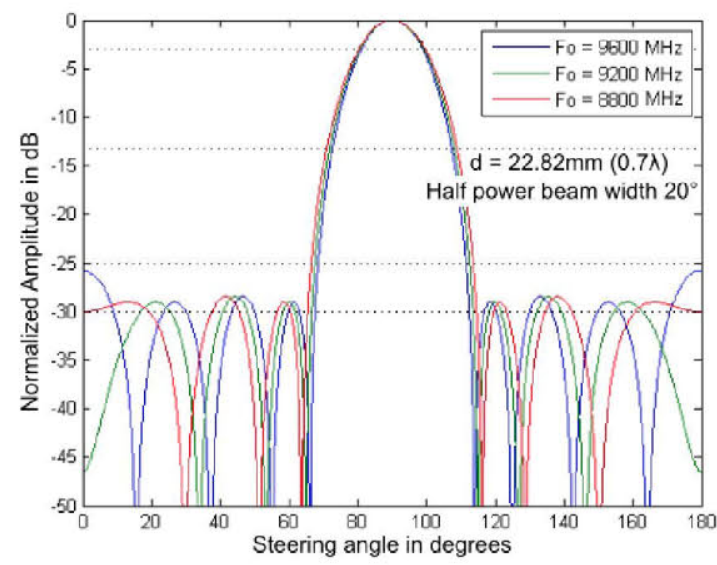

(b)

Fig. 1: Frequency Comparison Array Factor. (a): Azimuth. (b): Elevation.

degradation is acceptable keeping the side lobes level low. Further comparisons and specifications for other windows can be consulted on [2].

Other behaviors could be expected in the frequency band extremes and maximum steering angles, however these considerations are taken into account in order to maintain the system performance properly. In Fig. 1 a comparison between the center frequency and extremes frequency band for azimuthal and elevation array factor is presented, as well a comparison between two different steering angles, $45^{\circ}$ and $135^{\circ}$, at the center, and extreme frequency of the operating band is presented in Fig. 2. It is possible to appreciate that even at the extreme frequencies and for different steering angles, the design specifications are still accomplished.

A large number of elements per row is obtained (144), so it is important to reduce the distribution network complexity. Ideally, in order to achieve a good performance with the Taylor weighting, each element should receive the appropriate power percentage and diminish gradually between adjacent elements. However, this could complicate the power dividers design, for this reason it is important to implement a power sub array configuration. The power delivered to each sub array follows a similar Taylor weighting but keeping constant the power

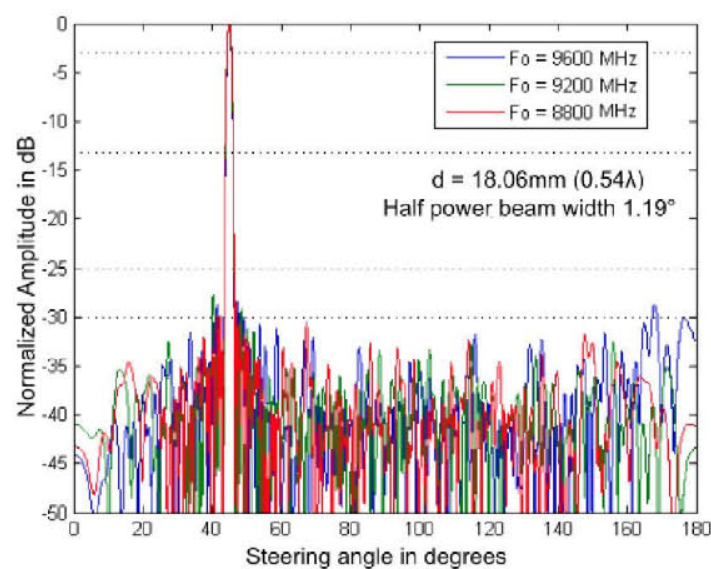

(a)

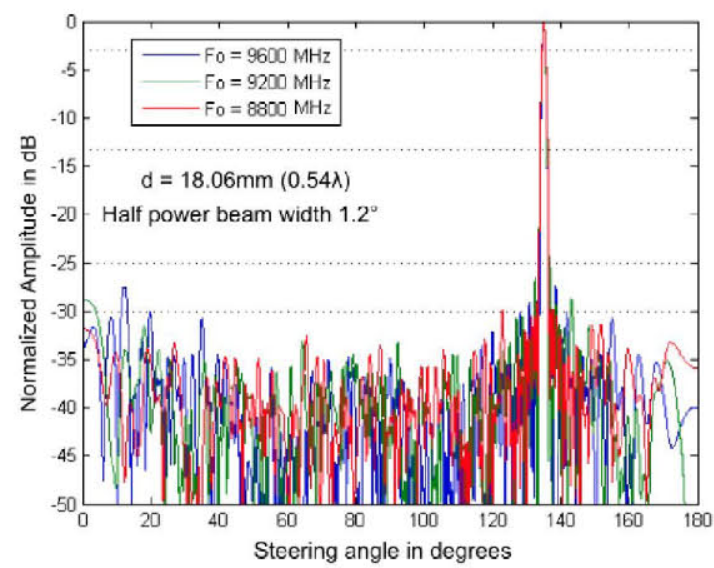

(b)

Fig. 2: Steering Comparison Array Factor. (a): Azimuth $45^{\circ}$. (b): Azimuth $135^{\circ}$.

delivered to each element of sub array as it is presented in Fig. 3.

It is important to take into account the quantification error produced by the implemented phase shifters as well. This effect is generated due to the limited number of phases of this devices as is defined in [3]. The first effect perceived is a gain loss at the main lobe, the second is the rise of undesirable quantification side lobes and the third is a pointing deviation in the main beam. This errors depends on the phase shifter number of bits $(B)$ and the half power beamwidth $\left(\theta_{-3 d B}\right)$. A simulation is performed to evidence the mentioned effects, with 4 bits phase shifter the side lobes level is below $-25 d B$ as is presented in Fig. 4, enough condition in order to accomplish the design specifications.

The conventional phased array architecture contemplates one phase shifter per radiating element, thereby the steering is controlled by changing the phase shift between adjacent radiating elements. However, this model could be expensive for large arrays, increasing the total price of the antenna. An alternative architecture have been proposed in [4], it uses one phase shifter per radiating sub-array, preserving the distance $d$ between adjacent radiating elements. The phase in each sub- 


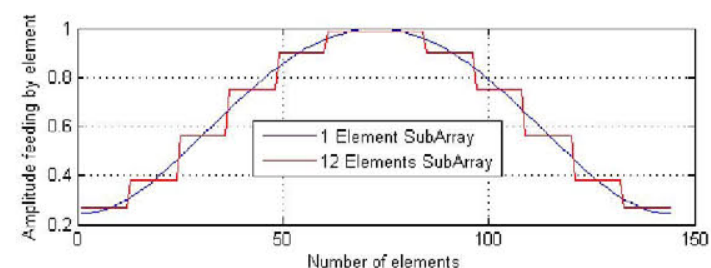

(a)

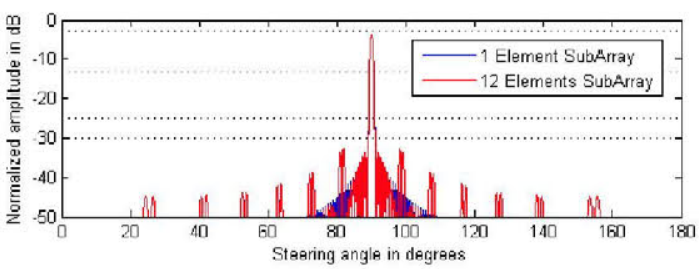

(b)

Fig. 3: Amplitude Sub Array Effect. (a): Amplitude Weighting. (b): Azimuth Array Factor.

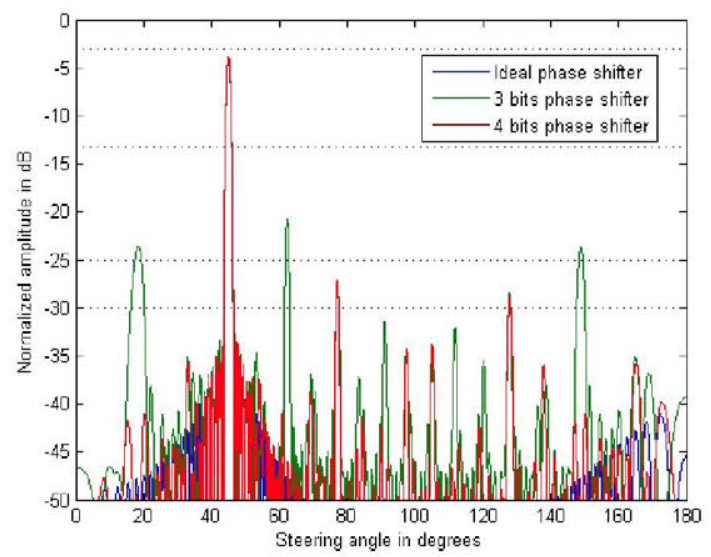

Fig. 4: Azimuth Array Factor with Phase Shift Quantification Error.

array is constant and the steering is controlled by changing the phase shift between adjacent sub-arrays. Unfortunately, this technique drastically increase the side lobes level, a sensitive parameter for radar applications as is depicted in Fig. 5. Nowadays, this situation has not been solved in its totality and is under investigation. In order to accomplish the side lobe levels requirement for the present design, one phase shifter per radiating element is used.

\section{RADIATING ELEMENT DESIGN}

The dipole is a well known antennas array element due to its feasible behavior and fabrication facilities, it can be manufactured by wire or printed technologies. A folded printed dipole is chosen as the radiating element for this design, it consists on two interconnected arms, one of them is directly connected to the feeding. The input impedance of the folded dipole is four times higher and presents higher frequency bandwidth than the conventional one, the arms width control the impedance adjustment [5]. The folded dipole model is implemented, simulated and optimized on CST Studio Suite

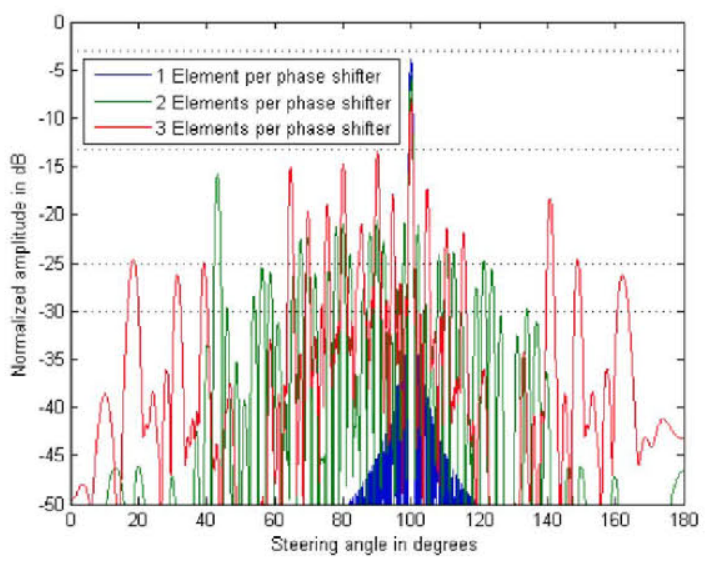

Fig. 5: Phase Sub Array Error Effect.

software over a Rogers substrate (RO4350B) with properties detailed in table III. In order to achieve $50 \Omega$ impedance coupling at the antenna input it is necessary to use a microstrip balun element defined in [6] as shown in Fig. 6.

TABLE III: RO4350B SUBSTRATE PROPERTIES.

\begin{tabular}{|c|c|}
\hline Parameter & Values \\
\hline \hline Dielectric Constant $\left(\varepsilon_{r}\right)$ & 3.66 \\
\hline Substrate Height $(\mathrm{H})$ & $0.5 \mathrm{~mm}$ \\
\hline Copper Thickness $(\mathrm{T})$ & $0.018 \mathrm{~mm}$ \\
\hline Loss Tangent & $0.0037 @ 10 \mathrm{GHz}$ \\
\hline Ground Plate Thickness $\left(T_{G}\right)$ & $2 \mathrm{~mm}$ \\
\hline
\end{tabular}

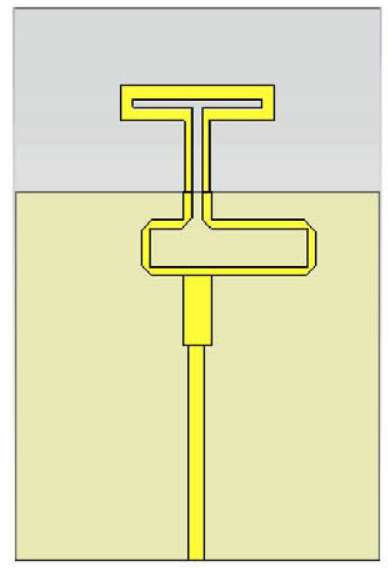

(a)

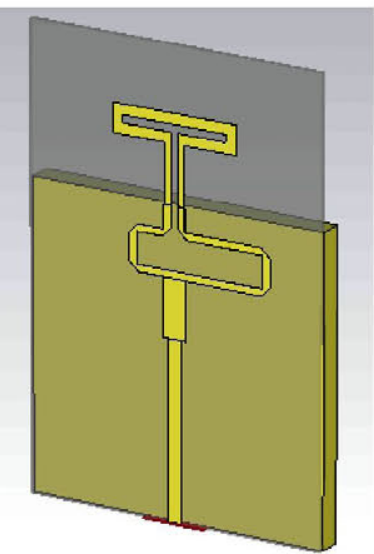

(b)
Fig. 6: Element Model. (a): Frontal View. (b): Perspective View.

The balun and the folded dipole are jointly simulated and optimized in order to obtain an appropriate impedance coupling over the frequency bandwidth keeping a stable radiation pattern. The S11 coefficient is below $-20 d B$ over all the frequency bandwidth as is depicted in Fig. 7, both element resonances can be noted as is expected. 


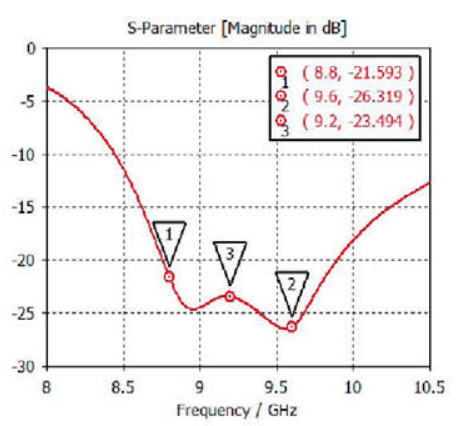

(a)

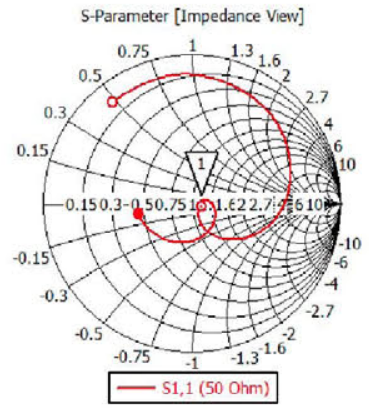

(b)
Fig. 7: Element Model Impedance Coupling. (a): S11 dB. (b): Zin.

The azimuth and elevation simulated radiation patterns at $9.2 \mathrm{GHz}$ are presented in Fig. 8, a maximum broadside gain of $4.71 \mathrm{dBi}$ is achieved. Finally, a four elements sub array prototype with uniform amplitude feeding is simulated in order to verify and validate the correct modeling as depicted in Fig. 9.

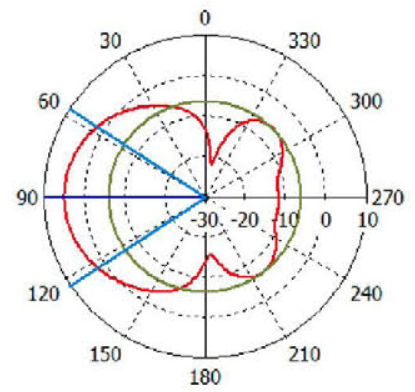

Phi / Degree vs. dB

(a)

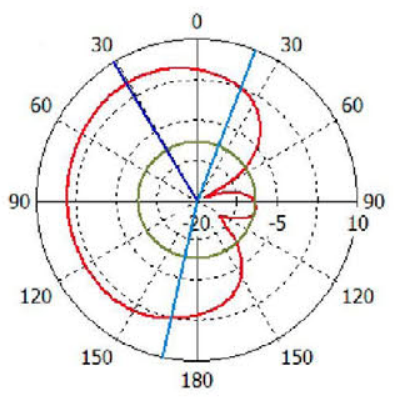

Theta / Degree vs. dBi

(b)
Fig. 8: Element Model Radiation Pattern. (a): Azimuth. (b): Elevation.

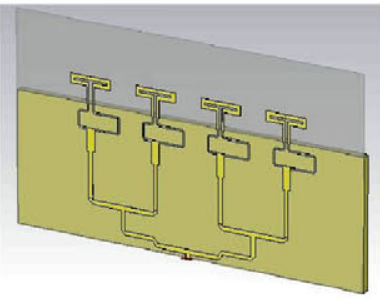

(a)

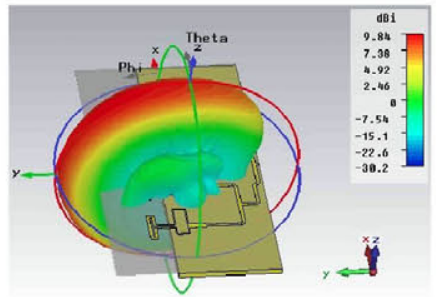

(b)
Fig. 9: Sub array Model. (a): Perspective View. (b): 3D Radiation Pattern

\section{Distribution Network DESIGN}

The distribution network is designed in order to achieve the chosen Taylor weighting with the purpose of side lobes reduction. First, the number of elements is established, the array consists of 5 rows with 144 elements as it is mentioned before. Therefore, it is necessary to design the complete distribution network for one row, 144 elements, and the complete distribution network to interconnect the five rows. Then, the distribution architecture is established using 2-way and 3-way power dividers. Finally, the power distribution is calculated and each power divider is designed, simulated, optimized and integrated using microstrip technology over the Rogers substrate (RO4350B) also used for the element design.

\section{A. Elements Distribution Network}

The elements distribution network is designed using 12 uniform power sub arrays, 4 asymmetric 3 -way power dividers, 2 asymmetric 2 -way power dividers and 1 symmetric power divider as it is presented in Fig. 10. It is necessary to adjust and optimize the physical parameters of power dividers to achieve the appropriate impedance value at the input and output ports $50 \Omega$.

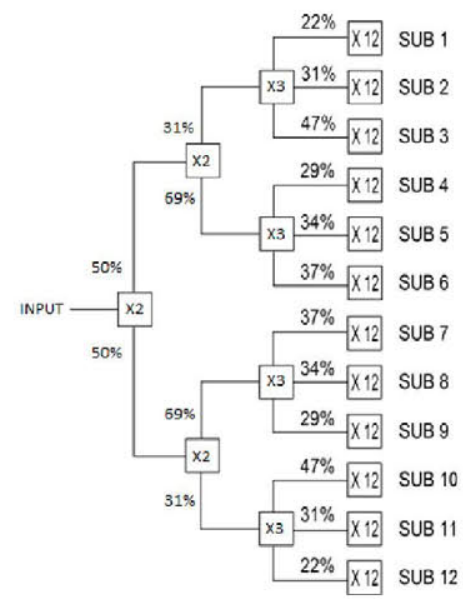

Fig. 10: Elements Distribution Network Architecture.

The designed power dividers and radiating elements are integrated on CST Studio Design tool. The complete rows dimensions are presented in Fig. 11, it is obtained a $16.3 \mathrm{~cm}$ length, $259.6 \mathrm{~cm}$ width and $2.5 \mathrm{~mm}$ height. The simulation results are shown in figure 12 , the port impedance is $50.125 \Omega$ at $9.2 \mathrm{GHz}$ and the $\mathrm{S} 11$ coefficient is below $-20 \mathrm{~dB}$ over all the frequency band.

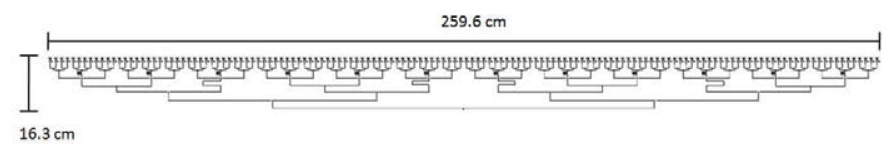

Fig. 11: Row Final Dimensions.

\section{B. Rows Distribution Network}

The process design is the same as previous section, the numbers of rows is established as 5 in order to achieve the elevation half power beamwidth $\left(\theta_{-3 d B}\right)$ specification $\left(20^{\circ}-25^{\circ}\right)$. It is necessary to use Taylor amplitude weighting in order to decrease the side lobes level as well. The distribution network is modeled, simulated and optimized on CST Studio Design tool over the same substrate, it is based on the 2-way and 3-way Wilkinson dividers architecture. The designed rows distribution network complete dimensions are presented in 


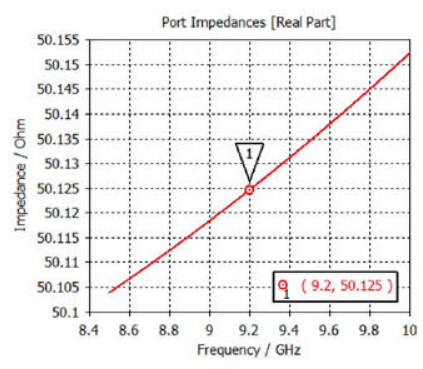

(a)

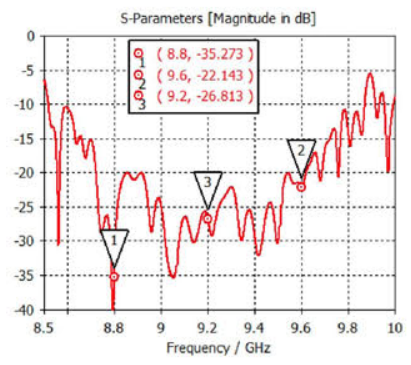

(b)
Fig. 12: Row Model Results. (a): Zin (b): S11 dB

Fig. 13. Finally, the simulation results are shown in Fig. 14, the ports impedance are equal to $50 \Omega$ at $9.2 \mathrm{GHz}$, the $\mathrm{S} 11$ coefficient is below $-20 d B$ over all the frequency band and the transmission coefficients accomplish the power distribution percentage.

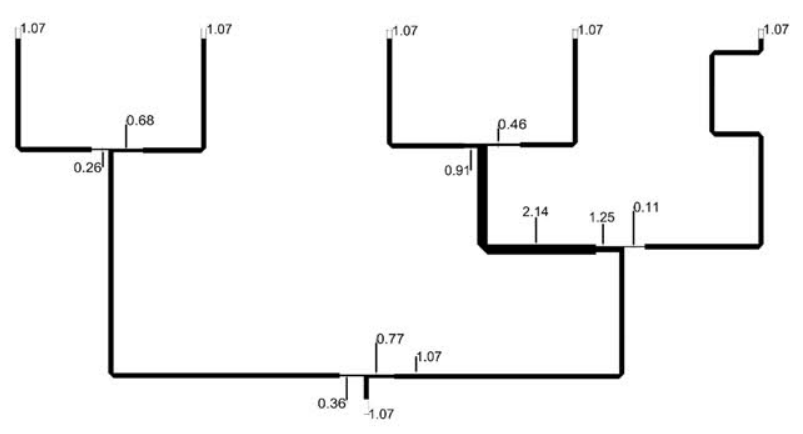

Fig. 13: Rows Distribution Network Dimensions [mm].

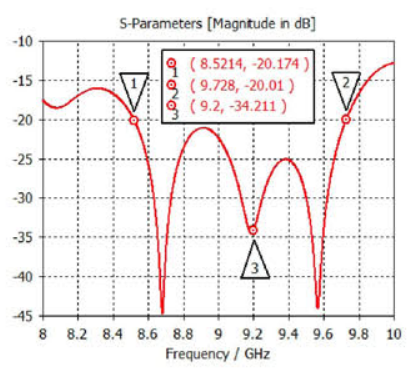

(a)

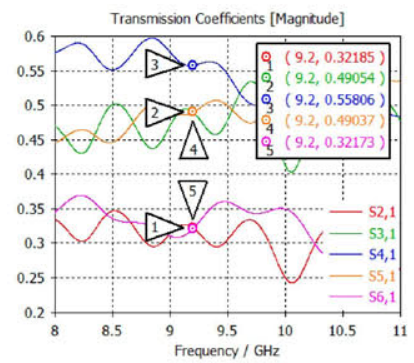

(b)
Fig. 14: Rows Distribution Network Results. (a): S11 dB. (b):Tx Coefficients

\section{CONCLUSION}

An appropriate design methodology for a phased array antenna in $\mathrm{X}$ band $(8.8-9.6 \mathrm{GHz})$ for radar applications is presented. In the first design stage is taken into consideration the specified parameters to be performed by the radiant system, the array number of elements and its separation are fixed. It is necessary to use a Taylor amplitude weighting in order to achieve the required side lobe ratio (SLR) design specifications, a SLR above $25 d B$ is obtained. When carrying out the amplitude weighting selection, the feeding network complexity is reduced by implementing amplitude sub arrays $(12 \cdot 12=144)$ keeping stable the parameters performance. The possibility of phase sub arrays grouping is explored as a strategy to reduce the required phase shifters number for the complete array, however the problems associated with these techniques are not resolved yet and remains under worldwide investigation. A printed folded dipole is chosen as the array radiating element due to its simplicity design, low cost, fabrication facilities and feasible integration with electronic circuits. The element is simulated and optimized in order to have an appropriate input impedance coupling, a gain of $4.71 \mathrm{dBi}$ is achieved. The distribution network is designed to accomplish the chosen amplitude weighting using 2-way and 3-way power dividers, it is simulated, optimized and integrated to the radiating elements using microstrip technology over the Rogers substrate (RO4350B), an appropriate impedance coupling is achieved over all the frequency band. Prototype construction and measured results will be presented in EuCap'2016 Conference in Davos, Switzerland.

\section{ACKNOWLEDGMENT}

The authors would like to express gratitude to UPM (Universidad Politécnica de Madrid), specially to the Radiation Group (GR) for the installations access, resources, the knowledge and time shared with us. Likewise to Colombian Government, CODALTEC (Corporación de Alta Tecnología para la Defensa) and GIDS (Grupo de Investigación y Desarrollo de Sensores) for all the support. The authors want to acknowledge as well the Spanish Government, Ministry of Economy, National Program of Research, Development and Innovation for the support of the project ENABLING5G "Enabling Innovative Radio Technologies for 5G networks" (Project number TEC2014-55735-C3-1-R) and the project Spacer Debris Radar (Radar para la detección de basura espacial) from Madrid Region Government (Project number S2013/ICE-3000 SPADERADAR-CM).

\section{REFERENCES}

[1] D. W. P. FENN. Alan J, TEMME. Donald H and C. W. E, "The development of phased-array radar technology," Lincoln Laboratory Journal, vol. 12 , no. 2 , pp. $321-340,2000$.

[2] F. Harris, "On the use of windows for harmonic analysis with the discrete fourier transform," Proceedings of the IEEE, vol. 66, no. 1, pp. 51-83, Jan 1978.

[3] T. A. Milligan, Modern Antenna Design, 2nd ed. Wiley-Interscience, 2005.

[4] T. Takano and K. Saegusa, "Analysis of a large phased array antenna in assembly of sub-arrays attached with phase shifters," in Antennas and Propagation Society International Symposium (APSURSI), 2013 IEEE, July 2013, pp. 286-287.

[5] L. d. H. A. Manuel Sierra Castañer, J.L Besada Sanmartín, Radiación y Propagación, U. P. de Madrid, Ed. ETSI Telecomunicación, Septiembre 2004.

[6] B. C. Wadell, Transmission Line Design Handbook. Artech House, 1991 